\author{
D. BHARATHI MOHAN ${ }^{1}$ \\ K. SREEJITH ${ }^{2}$ \\ C.S. SUNANDANA ${ }^{3, \infty}$
}

\section{Surface plasmon-exciton transition in ultra-thin silver and silver iodide films}

\author{
${ }^{1}$ Department of Mechanical Engineering, University of Coimbra, Coimbra, Portugal \\ 2 Institute for Materials Research, University of Salford, Salford, M5 4WT, UK \\ ${ }^{3}$ School of Physics, University of Hyderabad, Hyderabad 500 046, India
}

Received: 23 March 2007/Revised version: 31 July 2007

Published online: 29 August 2007 • () Springer-Verlag 2007

ABSTRACT Silver thin films in the thickness range $2-10 \mathrm{~nm}$ produced by thermal evaporation onto glass substrates were systematically iodized and carefully characterized by X-ray diffraction, atomic force microscopy (AFM) and optical absorption spectroscopy. While the uniodized films are X-ray amorphous in keeping with their quasi-continuous nature and 2D islanded structure, briefly iodized films showed characteristic beta AgI structure. Most interestingly, AFM of Ag films revealed uniform triangle-shaped embryos whose shape does not change appreciably upon iodization. Optical absorption spectra of uniodized Ag films show intense surface plasmon resonance (SPR) features with maxima at 440, 484 and $498 \mathrm{~nm}$ for the films of thicknesses 2, 5 and $10 \mathrm{~nm}$, respectively, with $5 \mathrm{~nm}$ films showing properties characteristic of optimally matched dielectric and electronic properties of the substrate and sample, respectively. Finally, an interesting and unique SPR-exciton phase transition is observed as the ultra-thin films are progressively iodized. These Ag and AgI films could be promising candidates for plasmonic and nanophotonic applications.

PACS 78.66.-w; 73.20.Mf; 71.35.Cc; 42.70; 68.37.Ps; 42.82.-m

\section{Introduction}

Nanostructured noble metal films (e.g. Ag) with thicknesses $\leq 10 \mathrm{~nm}$ support plasmon oscillations. Such films are discontinuous and consist of isolated islands each of nanoscale diameter involving large surface to volume ratio of crystallites constituting the film. These structures could therefore be modelled as 2D nanoisland lattices or as 2D photonic lattices of voids separating the islands. Electrons in metals form a dense fermion gas with strong electron-electron interaction. Virtually all electrons are simultaneously involved in the Coulomb interaction. Both individual and collective behaviour is exhibited by these electrons. For electromagnetic wavelengths larger than the Debye length, all electrons collectively behave as a uniform elastic medium showing organized longitudinal oscillations. Behaving as bosons, these so-called 'plasmon' oscillations can be created on the surface of metal films by external electromagnetic/electric fields propagating

Fax: +91-40-23010227, E-mail: csssp@uohyd.ernet.in along the surface. Behaviour of electrons is as individual particles for wavelengths less than the Debye length, in which case a screened Coulomb interaction prevails. The interaction of light with metal surfaces is strongly enhanced when the surface was patterned on a wavelength scale. Nanostructures supporting localized plasmons are subject to intense research for their applications in plasmonics including nanophotonic devices [1], chemical sensors [2], optical filters [3] and lefthanded materials [4]. These surface plasmon resonances in thin films [5] largely depend on the size, shape, metallic material and the surrounding dielectric matrix. Brief iodization of these ultra-thin metal films causes a controlled depletion of electron density leading to a gradual disappearance of plasmons and a progressive buildup of excitons and valence band structure of AgI. The decay of plasmons in $\mathrm{Ag}$ is apparently closely connected with the buildup of electron-hole pairs in $\mathrm{AgI}$ as found in our recent iodization experiments [6,7]. In this paper we discuss experimental results on plasmon formation in ultra-thin $\mathrm{Ag}$ films and the plasmon-exciton transition when subjected to electromagnetic radiation in progressively iodized Ag films. An area of fundamental scientific interest, and possible future applications, would be the study of the optical properties of discontinuous metal films in the presence of electric fields and currents applied to the films. A large surface to volume ratio is the property which might be exploited in devices intended to monitor variables that can be detected by surface defects, such as conductivity modulation due to gaseous component adsorption [8].

\section{$2 \quad$ Experimental techniques}

Portions of highly pure $\mathrm{Ag}$ (NFC, 99.9\%) were placed in a molybdenum boat and thermally evaporated on to the substrates kept at ambient temperature. The glass substrates were kept at a distance of $25-26 \mathrm{~cm}$ from the source for deposition. Prior to the deposition these substrates were cleaned as follows: the substrates were first immersed in boiling $10 \%$ soap solution with $90 \%$ water, rubbed with cotton in cold water to remove weathering, kept in chromic acid up to the boiling point for removing organic contaminants, washed in cold water to remove surface contaminants followed by ultra-sonification in iso-propyl alcohol for 3-5 min duration and, finally, these substrates were dried in air before loading 


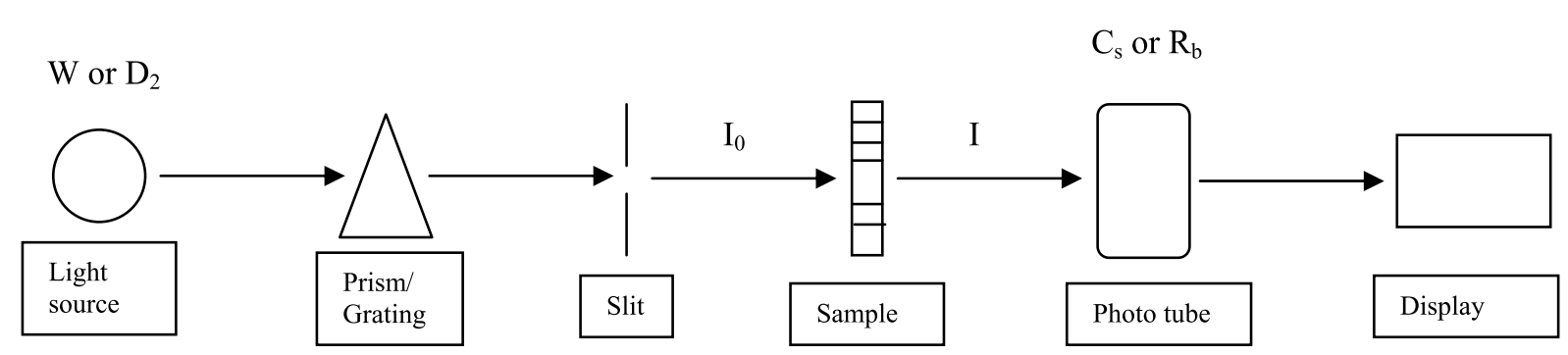

FIGURE 1 The schematic of optical absorption measurements setup for as-deposited silver and iodized Ag films

into the system for deposition. Pure silver films with thicknesses of 2, 5 and $10 \mathrm{~nm}$ were prepared at room temperature.

To iodize $\mathrm{Ag}$ films, a glass chamber was fabricated with dimensions of $10 \mathrm{~cm}$ height $\times 6 \mathrm{~cm}$ diameter. Iodine kept at the bottom of the lower half of the chamber sublimates at room temperature and slowly deposits on the Ag films kept at the top of the chamber. Thus, iodization was carried out for selected durations in the range 5-30 min. These films were characterized by X-ray diffraction $(\mathrm{XRD})$ using a Philips $\mathrm{X}$-ray powder diffractometer with $\mathrm{Cu} K_{\alpha}(\lambda=1.54056 \AA)$ radiation. To analyse the surface morphology, the films were examined by a SPA 400 atomic force microscope (AFM) using non-contact dynamic force mode. A Shimadzu UV-3101 spectrophotometer was used for absorption studies at $300 \mathrm{~K}$ in the UV-vis range from 300 to $700 \mathrm{~nm}$ with scanning rate $4 \mathrm{~nm} / \mathrm{s}$. The schematic of the optical absorption measurements setup for as-deposited silver and iodized silver films is given in Fig. 1.

\section{$3 \quad$ Crystal structure}

Ag films of thicknesses 2, 5 and $10 \mathrm{~nm}$ as evaporated on glass and progressively iodized were characterized by XRD as shown in Fig. 2. XRD patterns show quasiamorphous structure due to the nanocrystalline nature of silver particles. When subjected to a controlled flux of iodine vapours realized in a figure of eight or hourglass jig with a 1-mm opening at the centre for brief durations $\sim$ minutes, silver nanoparticles are gradually converted into AgI nanoparticles with predominant phase of $\beta$ - $\mathrm{AgI}$ (wurtzite structure) perhaps due to iodine excess $[(\mathrm{Ag} / \mathrm{I})<1]$ of silver iodide nanoparticles. This is something unusual and rare in ultra-thin films because in our previous study [7] it has been observed that the zincblende structure of silver iodide is stable in rf sputtered films. From these observations one would under- stand that the formation of $\gamma$-AgI nanoparticles is not reproducible on ultra-thin films, suggesting that both zincblende and wurtzite crystal structure are certainly possible as these films are grown on discontinuous silver films involving especially very large surface to volume ratio. Two important problems of discontinuous films are instability and poor reproducibility. It is also important here to observe that the enhancement in the intensity of XRD peaks with increasing film thickness is probably due to a decrease in the surface area of Ag nanoparticles.

\section{$4 \quad$ Surface morphology}

We used AFM to investigate the surface topography of as-deposited ultra-thin silver films and the growth of AgI nanoparticles upon increasing iodization time. A set of AFM images with $1 \mu \mathrm{m} \times 1 \mu \mathrm{m}$ field of view are shown in Fig. 3 for 'as-deposited' $\mathrm{Ag}$ and 30 min iodized films of two different thicknesses 2 and $5 \mathrm{~nm}$. A careful comparison of the as-deposited silver films reveals triangle-shaped embryos, a very interesting and unusual result in thermally evaporated films. 2-nm uniodized silver films show particle sizes of about $120 \mathrm{~nm}$. There are no changes observed in the shape and size with increasing film thickness to $5 \mathrm{~nm}$; however, at the same time the packing density of silver nanotriangles is very large. Iodization of these silver nanotriangles retains the shape with increasing particle size, which offers potential device possibilities.

\section{$5 \quad$ Optical properties}

The as-deposited silver films exhibit a broad surface plasmon resonance (SPR) with peak maxima at 440 , 484 and $498 \mathrm{~nm}$ for the films of thicknesses 2, 5 and $10 \mathrm{~nm}$, respectively, whereas SPR is diminished for a 20 -nm-thick

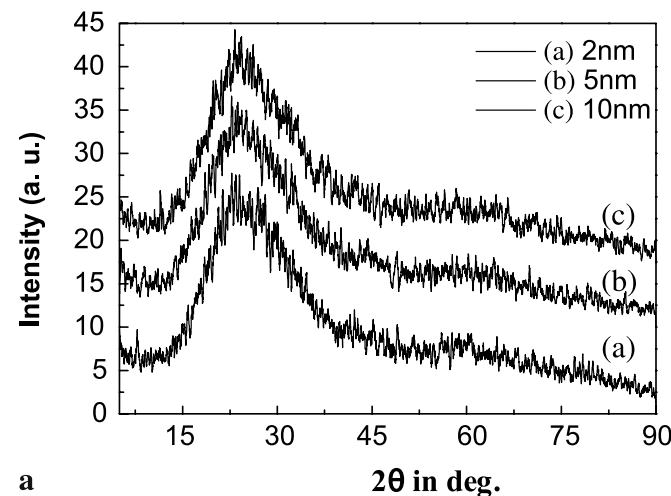

$\mathbf{a}$

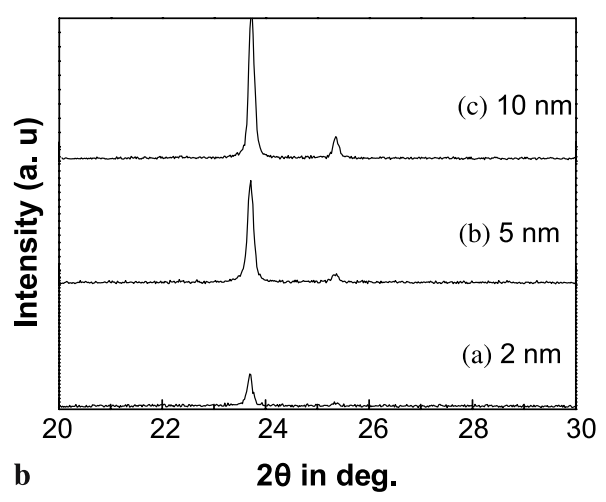

FIGURE 2 X-ray diffractograms of (a) as-deposited $\mathrm{Ag}$ films and (b) films iodized for $30 \mathrm{~min}$ of different thicknesses 2, 5 and $10 \mathrm{~nm}$ 


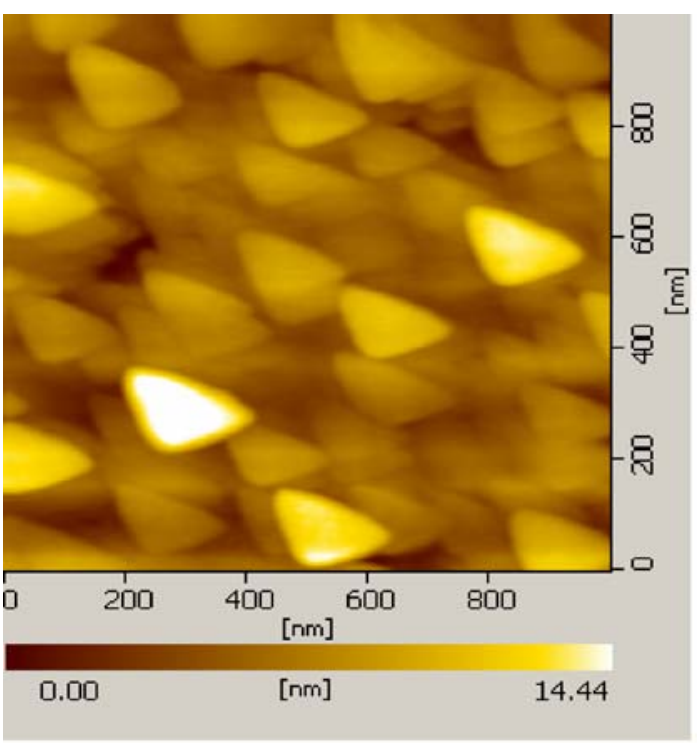

a

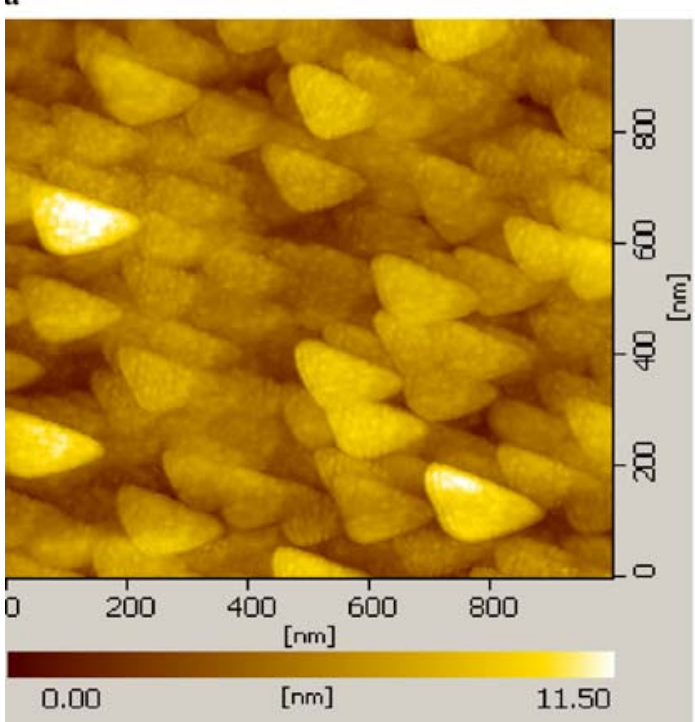

c $(\mathbf{c}, \mathbf{d})$ as-deposited silver and iodized films of thickness $5 \mathrm{~nm}$

film. SPR arises from the collective excitation of valence electrons from Ag nanoparticles whose densities are finite and strongly size dependent. The wavelength of the SPR on metallic films depends on many important factors involving shape, size, dispersion, surface roughness and dielectric constant of the matrix (e.g. glass) on which the Ag nanoparticles were deposited. The line width (FWHM) of the plasmon resonance is very large $(\sim 165 \mathrm{~nm})$ and it is controlled by processes such as crystallite/cluster shape fluctuations. In addition, clustermatrix interaction can also contribute to the line width. For silver particles containing more than 150 atoms, the optical measurements of Charle et al. [9] showed that the total line width of the plasmon peak scales with the inverse of the cluster diameter. In fact the 'composite' line width in such a case is the sum of several individual plasmon absorption lines, belonging to clusters of different sizes. SPR is red shifted by $0.32 \mathrm{eV}$ (from 2.81 to $2.49 \mathrm{eV}$ ) besides a doubled line width with increasing thickness of silver films. The above shift may be ascribed to an increased electromagnetic interaction between the $\mathrm{Ag}$ particles $[10,11]$ with the systematic decrease of the surface to volume ratio with increasing particle size [12] and of surface roughness with increasing film thickness [13]. Thus, it is clearly a case of electromagnetic absorption by small metal particles stabilized in a dielectric matrix - the matrix here being AgI. The intensity of the SPR tremendously increases for the 5-nm-thick film, suggesting that $5 \mathrm{~nm}$ could be a critical thickness with optimally matched dielectric and electronic properties of the substrate and the nanocomposite, respectively.

Upon controlled iodization, iodine vapour was deposited on the Ag surface and, by the action of several processes initiated by surface diffusion at the gas-solid interface, the silver nanoparticles' surfaces were eventually iodized. As the silver iodide begins to grow on the surface at the early stage of iodization $(5 \mathrm{~min})$ the core still remains as silver - constituting a $\mathrm{AgI} / \mathrm{Ag}$ core-shell type of growth. This type of 

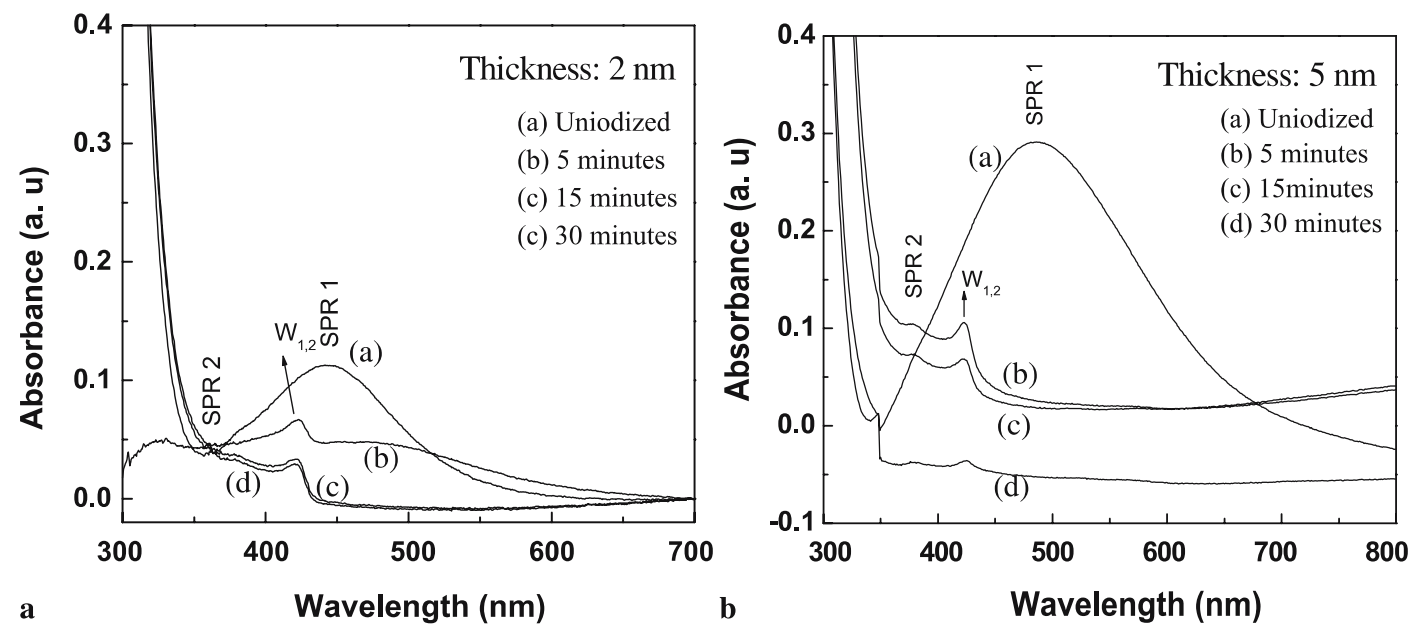

FIGURE 4 Optical absorbance spectra of asdeposited $\mathrm{Ag}$ and iodized films for periods ranging from $5 \mathrm{~min}$ to $30 \mathrm{~min}$
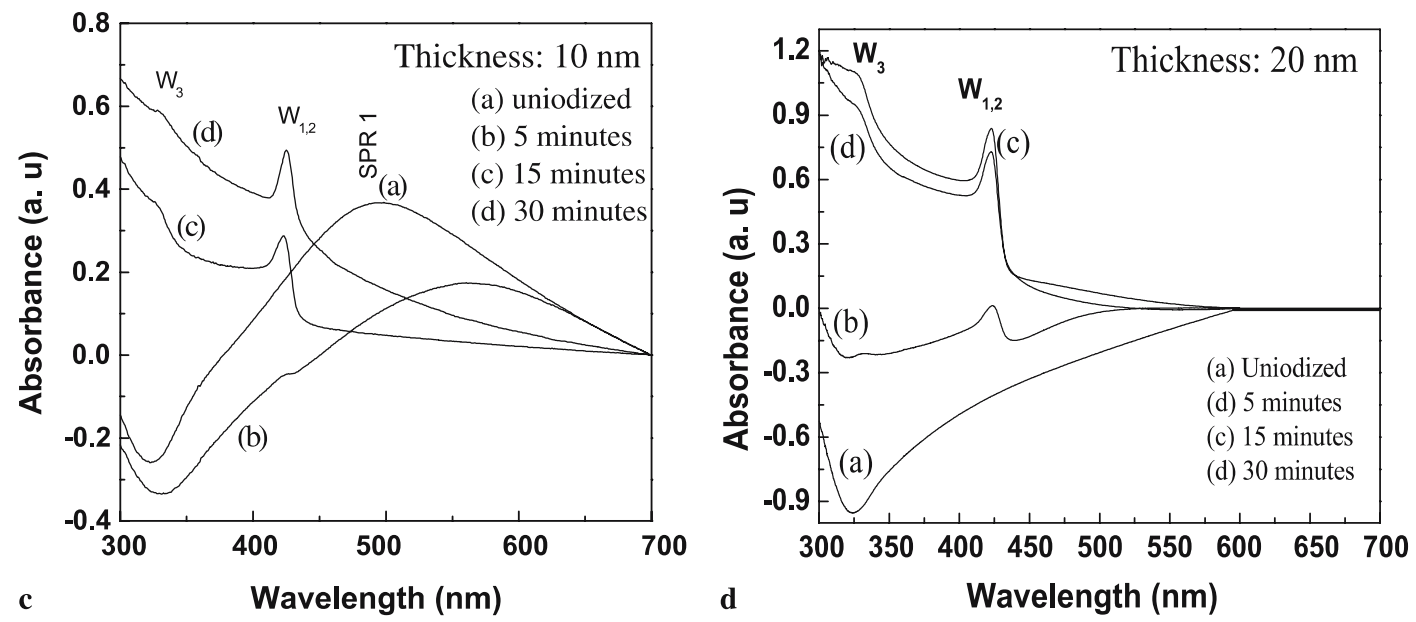

growth chiefly depends on the surface morphology of the film, rate of iodization, temperature at which iodization takes place and finally the film thickness. Figure 4 a shows optical absorption of a 2-nm-thick silver film revealing a weak SPR 1 with red shift after 5-min iodization. Additionally, two new peaks at $420 \mathrm{~nm}$ (classified as a $W_{1,2}$ exciton) and $380 \mathrm{~nm}$ (classified as SPR 2) are due to the formation of silver iodide on the surface as a shell type and an uniodized silver core, respectively. A core-shell type of growth is observed only by adopting the controlled iodization technique. After 30-min iodization, SPR 2 completely diminished as the silver core is depleted ensuring that the silver iodide growth is completed. At this juncture it would be appropriate to focus on the coupling between the Ag and AgI 'nanocomposite' features. Initially, there are only Ag (a)spherical nanoparticles. Upon iodization the conduction electrons of $\mathrm{Ag}$ are used to form $\mathrm{AgI}$ nuclei with I capturing an electron to form $\mathrm{I}^{-}$and eventually the $\mathrm{I}^{-}$molecular orbitals (MO) up to highest occupied molecular orbital (HOMO) and the valence band. The formation could be thought of in the I/AgI/Ag two-interface model [14]. Upon even 1-min exposure to I, the plasmon resonance of Ag would start diminishing and a AgI/Ag diffusion barrier (a distribution of barriers is possible) would be established. The diffusion is controlled by (a) the strength of the barrier, (b) the surface electron concentration on the Ag side and (c) the I concentration on the left-hand side of the AgI shell. As the electrons are continually depleted, the SPR intensity decreases and there is a corresponding buildup of excitons. The distribution of Ag nanoparticle sizes and shapes controls the diffusion kinetics.

The evolution of the optical absorption peak at $420 \mathrm{~nm}$ (due to the dipole-forbidden ${ }^{4} d_{10}-{ }^{4} d_{9} 5 s$ transition in Ag, allowed by the tetrahedral symmetry of the $\mathrm{Ag}^{+}$ion in the wurtzite AgI structure and the resultant $\mathrm{p}-\mathrm{d}$ hybridization) has been attributed to the $W_{1,2}$ exciton [15]. The 420-nm exciton peak, the sharp rise in absorption below $320 \mathrm{~nm}$ and the long wavelength tail due to the intrinsic Frenkel disorder all systematically characterize the very basic process of the band structure formation of AgI starting from the nanocluster level [16]. 2- and 5-nm-thick (ultra-thin) films exhibit an exciton at $420 \mathrm{~nm}$ with larger intensity after 5-min iodization; however, on further iodization (15- and 30-min iodization) the intensity declined and the peak was red shifted perhaps due to the larger size of silver iodide particles (Fig. 4a and b).

Ultra-thin films $(2-5 \mathrm{~nm})$ exhibit a sharp rise in absorption below $330 \mathrm{~nm}$ with very high intensity due to quantum confinement effects and it is resolved at $330 \mathrm{~nm}$ (attributed to $W_{3}$ ) with increasing film thickness to $10 \mathrm{~nm}$ for longer times of iodization. After 30-min iodization, SPR 2 completely disappeared and in the mean time a new peak developed near $330 \mathrm{~nm}$ due to the spin-orbit split $\mathrm{I}^{-}$valence of the spinorbit interaction attributed to the $W_{3}$ exciton. The top of the valence band $\left(\Gamma^{8}\right)$ is triply degenerate without spin-orbit interaction in the wurtzite structure. This degeneracy is reduced 
by spin-orbit splitting to a doubly degenerate state $\left(\Gamma_{7}\right)$ and a singlet $\left(\Gamma_{9}\right)$. The $\Gamma_{9}$ doublet gives the $W_{1,2}$ peak whose degeneracy is lifted due to some strain field change at the crystallite surface (for example, surface reconstruction or surface defects) [5, 6]. A 20-nm-thick film of silver shows strong exciton peaks upon increasing iodization time, corroborating the formation of a large number of excitons (Fig. 4d). From these results one would possibly say that the better range/thickness to work for the formation of the SPR could be 2 to $10 \mathrm{~nm}$. These results agree with our earlier studies on rf sputtered films where the valence band degeneracy is lifted at room temperature for 20- and 30-nm-thick films. Optical absorption of vapour quenched metastable $\mathrm{Ag}-\mathrm{Cu}$ thin films revealed that the strain induced size control by $\mathrm{Cu}$ helps in the confinement of excitons in AgI nanoparticles [17]. Mshvelidse et al. studied excitonic absorption of silver iodide quantum dots in an aluminoborosilicate host network structure at $10 \mathrm{~K}$ [18]. Mochizuki et al. measured optical absorption of AgI at temperatures from $7 \mathrm{~K}$, through the superionic phase transition point $T_{\mathrm{c}}(419 \mathrm{~K})$, to $472 \mathrm{~K}$ [19].

Band gap energy is determined from the expression of the semiconductor with a direct band gap, $(\alpha h v)^{2}=B\left(h v-E_{\mathrm{g}}\right)$, where $\alpha$ is the absorbance coefficient, $h v$ is the energy of incident light, $B$ is a parameter that depends on the interband transition probability and $E_{\mathrm{g}}$ is the energy gap. The plot of $(\alpha h v)^{2}$ vs. incident photon energy $h v$ is found to be a straight line. The intercepts of the linear plots on the energy axis at $(\alpha h \nu)^{2}=0$ gave a band gap value determined for both $W_{1,2}$ and $W_{3}$ excitons. 2-, 5- and 10-nm-thick silver films show band gaps of 2.85, 2.82, 2.77 and $2.70 \mathrm{eV}$ after 30 -min iodization, respectively. The band gap decreases with increasing film thickness, indicating the effect of increasing particle size and correspondingly decreasing quantum confinement effects. In addition, film stress tends to increase with thickness, causing band edge shifts.

\section{6}

\section{Conclusions}

Ultra-thin silver films $(2-10 \mathrm{~nm}$ thick) produced by thermal evaporation onto glass substrates were systematically iodized and carefully characterized by XRD, AFM and optical absorption spectroscopy. Uniodized films are Xray amorphous in keeping with their quasi-continuous nature and 2D islanded structure, while briefly iodized films showed characteristic beta AgI nanostructure developing gradually. Most interestingly, AFM of Ag films revealed triangle-shaped embryos whose shape does not change appreciably upon iodization. Optical absorption spectra of uniodized Ag films show intense surface plasmon resonance (SPR) features with maxima at 440, 484 and $498 \mathrm{~nm}$ for the films of thicknesses 2, 5 and $10 \mathrm{~nm}$, respectively, with 5-nm films showing properties characteristic of optimally matched dielectric and electronic properties of the substrate and sample, respectively. Finally, an interesting and unique SPR-exciton phase transition is observed as the ultra-thin films are progressively iodized. These Ag and AgI films could be promising candidates for plasmonic and nanophotonic applications.

ACKNOWLEDGEMENTS Sincere thanks are due to the University of Hyderabad for the award of a research fellowship to D. Bharathi Mohan under the UPE programme. K. Sreejith thanks the University of Hyderabad for financial support during this project.

\section{REFERENCES}

1 L. Hesselink, in Photonics 2006, 8th Int. Conf. Optoelectronics, Fiber Optics and Photonics, Hyderabad, India, 13-16 December 2006, Abstracts 2, p. 241

2 J.J. Storhoff, R. Elghanian, R.C. Mucic, C.A. Mirkin, R.L. Letsinger, J. Am. Chem. Soc. 120, 1959 (2002)

3 Y. Dirix, C. Bastiaansen, W. Smith, Adv. Mater. 11, 223 (1999)

4 J.B. Pandry, Phys. Rev. Lett. 85, 3966 (2000)

5 E.N. Ekonomou, Phys. Rev. 182, 539 (1969)

6 D. Bharathi Mohan, C.S. Sunandana, J. Appl. Phys. 100, 064314 (2006)

7 D. Bharathi Mohan, V. Sivaji Reddy, C.S. Sunandana, Appl. Phys. A 86, 73 (2007)

8 Z.H. Meiksin, Phys. Thin Films 8, 161 (1975)

9 K.P. Charle, W. Schulze, B. Winter, Z. Phys. 12, 471 (1989)

10 C. Francisco, Phys. Rev. B 25, 7875 (1982)

11 L. Genzel, T.P. Martin, Surf. Sci. 34, 33 (1973)

12 F. Moresco, M. Rocca, T. Hildebrandt, M. Henzler, Phys. Rev. Lett. 83, 2238 (1999)

13 K. Kurihara, C. Rockstuhi, T. Kano, T. Arai, J. Tominaga, Nanotechnology 16, 1565 (2005)

14 P. Senthil Kumar, C.S. Sunandana, Proc. SPIE 4807, 241 (2002)

15 M. Cardona, Phys. Rev. 129, 69 (1963)

16 P. Senthil Kumar, C.S. Sunandana, Nano Lett. 2, 975 (2002)

17 Y.Y. Gurevich, A.K. Ivanov-Shits, in Semiconductors and Semimetals, vol. 26, ed. by R.K. Willardson, A. Beer (Academic, New York, 1998), p. 230

18 G. Mshvelidse, O. Gogolin, E. Tsitshivili, C. Markle, J.S. Chadha, A. Hepting, F. Gindele, W. Petri, W. Send, D. Gerdhsen, U. Woggon, C. Klingshrin, Phys. Stat. Solidi B 207, 369 (1998)

19 S. Mochizuki, Y. Ohta, J. Luminesc. 87, 299 (2000) 